\title{
UMA AVALIAÇÃO DAS PUBLICAÇÕES EM LEALDADE DO CONSUMIDOR EM PERIÓDICOS BRASILEIROS ${ }^{1}$
}

\author{
AN EVALUATION OF PUBLICATIONS IN CONSUMER LOYALTY IN BRAZILIAN \\ JOURNALS
}

\section{EVALUACIÓN DE LAS PUBLICACIONES EN FIDELIDAD DE CONSUMIDOR EN REVISTAS BRASILEÑAS}

Tiago Rusin, Doutorando em Nutrição Humana pela Universidade de Brasília - UnB, Analista Ambiental do Ministério do Meio Ambiente (MMA). Endereço Profissional: Ministério do Meio Ambiente, Esplanada dos Ministérios, Bloco B, sala 744, Brasília, DF Brasil, CEP 70068900. Email: eng.rusin@ gmail.com.

Luiz Fernando Silva Pinto, Mestrando em Administração pela Universidade de Brasília UnB. Endereço Profissional: Campus Darcy Ribeiro - Prédio da FACE - Brasília Distrito Federal - CEP: 70910-900. Email: luizfernando4@gmail.com.

Luiz Medeiros de Araujo Neto, Mestre em Administração pela Universidade de Brasília UnB. Professor do Centro Universitário - UNIEURO. Endereço Profissional: Avenida das Nações, Trecho 0, Conjunto 05 - Brasília - DF. Email: medeiros2002@ hotmail.com.

Vivian Aline Carolino, Mestranda em Administração pela Universidade de Brasília - UnB. Endereço Profissional: Correios, Setor Bancário Norte, Quadra 01, Edifício Sede dos Correios, Brasília, DF - Brasil - CEP: 70040-010. vivianaline@ correios.com.br.

Gisela Demo, Pós-doutora em Management \& Organizations pela University of California, Los Angeles. Professora Associada do Programa de Pós-Graduação em Administração da Universidade de Brasília. Endereço Profissional: Campus Universitário Darcy Ribeiro, Prédio da FACE, Asa Norte, Brasília-DF, Brasil. CEP: 70910-900. Telefone: 55 (61) 3107-0759. Blog: gpegpc.blogspot.com. E-mail: giselademo@unb.br.

\section{RESUMO}

O artigo apresenta uma síntese da literatura e uma análise bibliométrica da produção nacional do campo de conhecimento constituído pelo conceito de lealdade ou fidelidade do consumidor, estudado nos últimos cinco anos relevantes à área da Administração. No total foram encontrados 42 artigos que atenderam aos critérios de busca em 27 revistas selecionadas. Os artigos foram tabulados e analisados de acordo com as seguintes categorias: nome do artigo, autores, Qualis Capes, ano de publicação, instituições participantes, enquadramento (teórico ou teórico-empírico), e, quando enquadrado como teórico-empírico: natureza do estudo, setor da economia, ramo de atuação, instrumentos de coleta de dados e técnicas de análise de dados. Verificou-se que existe uma predominância de pesquisas teórico-

\footnotetext{
${ }^{1}$ Artigo submetido em 28/02/2016, revisado em 04/04/2016, aceito em 10/09/2016 e divulgado em 30/12/2016 pelo Editor João Carlos Hipólito Bernardes do Nascimento, após double blind review.
}

GєCont, v. 3, n. 2, Floriano-PI, Jul-Dez. 2016. 
empíricas de natureza quantitativa sobre o tema. Os resultados permitiram identificar a crescente demanda de conhecimento sobre o tema da lealdade do consumidor, bem como lacunas na literatura que subsidiaram a proposição de uma agenda de pesquisa.

Palavras-chave: Lealdade; Fidelidade; Revisão Bibliométrica; Agenda de Pesquisa.

\section{ABSTRACT}

The article presents a synthesis of the literature and an analysis of national bibliometric knowledge production field consists of the loyalty and customer loyalty concepts studied in the past five years relevant to the area of administration. In total we found 42 articles that met the search criteria on 27 selected journals. Papers were tabulated and analyzed according to the following criteria:paper title, authors, journal name, quails ranking, year of publication, participating institutions, framework (theoretical or theoretical and empirical), and when framed as theoretical and empirical: nature of the study, sector of the economy, industry, instrument of data collection and data analysis techniques. It was found a predominance of quantitative theoretical and empirical research on the subject. The results showed the growing demand for knowledge on the subject of loyalty and customer loyalty, as well as gaps in the literature, that supported the proposition of a research agenda.

Keywords: Loyalty, Fidelity, Bibliometric Review, Research Agenda.

\section{RESUMEN}

El artículo presenta una síntesis de la literatura y un análisis del campo nacional de producción de conocimiento bibliométrico-biombolical consiste en los conceptos de lealtad y lealtad de clientes estudiados en los últimos cinco años relevantes para el área de administración. En total, hemos encontrado 42 artículos que cumplieron con los criterios de búsqueda en 27 revistas seleccionadas. Los trabajos se tabularon y analizaron de acuerdo con los siguientes criterios: título del artículo, autores, nombre de la revista, clasificación de las codornices, año de publicación, instituciones participantes, marco (teórico o teórico y empírico) y enmarcado como teórico y empírico: naturaleza del estudio, Sector de la economía, industria, instrumento de recolección de datos y técnicas de análisis de datos. Se encontró un predominio de la investigación teórica y empírica cuantitativa sobre el tema. Los resultados mostraron la creciente demanda de conocimiento sobre lealtad y lealtad de los clientes, así como brechas en la literatura, que apoyaron la propuesta de una agenda de investigación.

Palabras clave: Lealtad, Fidelidad, Revisión Bibliométrica, Agenda de Investigación.

\section{INTRODUÇÃO}

crescente oferta de informações modificou a forma como os setores da
economia se relacionam. A procura de novos nichos de mercado, juntamente
com a busca por soluções para problemas de relacionamento com os clientes, fizeram as empresas demandarem novas estratégias. Uma das novas estratégias é a compreensão da forma de pensar e interagir do consumidor, mais precisamente os mecanismos de desenvolvimento da lealdade e fidelidade à marca.

A lealdade e/ou fidelidade do consumidor, termos que serão utilizados como sinônimos no presente trabalho, em relação à determinada empresa, marca, produto ou serviço pode influenciar fortemente a sustentabilidade dos negócios. Assim, a literatura internacional tem procurado abranger uma vasta gama de análises que envolvam não só a lealdade no contexto de grandes varejistas, mas também na indústria de serviços, internet e redes sociais.

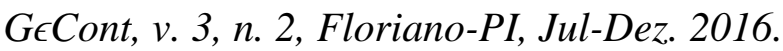


Nesse trabalho, tem-se como objetivo observar a literatura teórica nacional sobre o tema (estado da arte no Brasil). Ainda, uma revisão da produção brasileira (análise bibliométrica) dos últimos cinco anos sobre o tema lealdade do consumidor. O trabalho se justifica dado que os estudos brasileiros ainda continuam muito concentrados na área de varejo de produtos. Diante dessa realidade, fazem-se necessários estudos sobre lealdade que também considerem outros mercados e formas de consumo.

\section{ESTADO DA ARTE}

No contexto de marketing, derivado de conceitos trazidos por Zeithmal et al. (1996) e Sirdeshmukh et al. (2002), a lealdade pode ser definida como uma intenção de desempenhar um conjunto de comportamentos que sinalizem uma motivação de manter um relacionamento com determinada empresa.

Uncles et al. (1995) sugerem que o conceito de lealdade pode ser visto sob três formas. A lealdade como uma atitude que, por vezes, leva a um relacionamento com a marca, ou ainda, a lealdade expressa em termos de comportamentos revelados (isto é, o padrão de compras passadas) e, por fim, a compra moderada por características, circunstâncias e / ou a situação de compra do indivíduo.

O fato é que a necessidade de se cultivar a lealdade de consumidores tem sido colocada por gestores e autores como um dos mais importantes desafios a ser enfrentado pelas organizações (GREMLER e BROWN, 1999).

$\mathrm{Na}$ busca pelo melhor entendimento da lealdade, dentro da vertente da análise do comportamento do consumidor, a abordagem trazida pelo modelo BPM - Behavioral Perspective Model representa uma evolução dentro da tradição de interpretar o comportamento complexo (FOXALL et al., 2006). De acordo com este modelo, o comportamento do consumidor pode ser resumido em uma sequência de eventos que começa pelo desejo de consumir algo, seguido da busca interna e externa pelas alternativas para satisfazer tal desejo (quando ocorre na interseção de um cenário e da história de aprendizagem de um indivíduo de consumo), passa pela ação de comprar e chega à análise posterior do objeto em uso, avaliação esta que pode afetar a probabilidade de recompra e fidelização. No modelo, a história do consumidor vai sendo composta pelas consequências utilitárias (mediada pelo produto - benefício de uso que trouxe ao consumidor) e informativas (mediada por outras pessoas - imagem ou status trazido ao consumidor).

Cabe ressaltar que a lealdade do consumidor depende mais de motivações "emocionais" (comprometimento afetivo) do que de motivações "racionais" para continuar a relação (ČATER; ČATER, 2010).

Uma estratégia muito utilizada pelas organizações na tentativa de construir uma história positiva com os consumidores e aumentar sua lealdade é a adoção de programas de fidelização. Os programas adotados pelas companhias aéreas estiveram entre os primeiros na busca pela fidelização, sendo que o principal objetivo seria a alteração na frequência de compra e redução dos repertórios de marcas utilizadas por seus clientes (SHARP; SHARP, 1997).

Sharp e Sharp (1997) realizaram amplo estudo em uma grande empresa, o qual tratavase do maior programa de fidelização da Austrália e um dos maiores do mundo na época. Os resultados, no entanto, seguem uma tendência já discutida em estudos de comportamento de consumo, que afirmam ser muito difícil mudar o padrão de repetição de compra dos mercados. Apesar de inseguros sobre a efetividade dos programas de fidelidade, um grande número de varejistas ainda continua recorrendo a essa estratégia.

Abordagens mais recentes sugerem que não é esperado que o consumidor seja mais leal à marca, mas sim que compre muito volume de alguma marca em cada ocasião, mais do que de outras, uma vez que, independentemente dos esforços e estratégias adotadas pelas 
organizações, os consumidores demonstram um padrão de repertório multimarcas entre as ocasiões de compra (WELLS; FOXALL, 2013).

Menos voltados para consumo de produtos e mais para a indústria de serviços, Krystallis e Chrysochou (2014) confirmaram estudos anteriores que sugerem que a evidência da marca influencia significativamente a satisfação do consumidor, atitude e fidelidade comportamental também em relação às marcas de serviços. Além disso, ao contrário dos estudos anteriores, os elementos de comunicação controlada (ex: propaganda e promoções) não tiveram nenhuma influência na satisfação do consumidor com marcas de serviços. Apesar disso, os mesmos elementos de comunicação moldaram direta e significativamente as percepções do consumidor das várias dimensões da marca e suas atitudes gerais em relação à marca.

Alguns estudos relatam ainda o papel da satisfação do consumidor como gerador de fidelidade. Analisando a eficácia da customização de serviços, Coelho e Henseler (2012) encontraram que a customização aumenta a qualidade percebida do serviço, satisfação do consumidor, confiança e em último grau fidelidade do consumidor em relação a um provedor de serviço. Customização tem tanto efeitos diretos quanto mediados na fidelidade do consumidor e interage com os efeitos da satisfação do consumidor e confiança na fidelidade.

A satisfação do consumidor é um ingrediente essencial para a fidelidade ao serviço, mas existem mediadores entre satisfação e fidelidade. Comprometimento, confiança e envolvimento são cada um dos mediadores parciais entre satisfação e fidelidade, enquanto valor percebido é um mediador completo (CHEN; HU, 2012). Complementarmente, o estudo de Setó-Pamies (2012), que visou analisar o impacto da qualidade do serviço, satisfação e confiança na fidelidade do consumidor em relação ao provedor do serviço, revela como fidelidade depende do grau de satisfação e confiança, enfatizando a importância deste último para o processo.

Indo da preocupação com o consumo de produtos e passando pela observação da indústria de serviços, em estudos mais recentes é possível identificar a preocupação em entender o mundo virtual e seus impactos na lealdade dos consumidores, que em seu trabalho Toufaily et al. (2012) chamaram de "e-fidelidade".

Laroche et al. (2012), por exemplo, descobriram que as comunidades de marca estabelecidas em redes sociais têm efeito positivo nos marcadores da comunidade (ex: consciência compartilhada, rituais e tradições compartilhadas e obrigações para a sociedade), as quais têm efeito positivo nas práticas de criação de valor (ex: networking, engajamento com a comunidade, gerenciamento de impressões e uso da marca), o que, consequentemente, leva a uma maior fidelidade.

Casaló et al. (2010) desenvolveram estudo visando determinar os principais antecedentes e consequências do envolvimento do consumidor em comunidades online de software livre. Os achados dos autores demonstram que a satisfação com uma comunidade virtual pode aumentar o grau de participação do consumidor. Ao mesmo tempo,uma maior identificação com a comunidade virtual pode aumentar indiretamente a participação do consumidor graças ao melhoramento da sua satisfação com a comunidade. Também foram encontrados efeitos positivos e significativos da identificação do consumidor e participação no nível da promoção da comunidade. Finalmente, efeitos positivos e significativos da participação e satisfação com a comunidade em relação à fidelidade ao software livre também foram encontrados.

Torna-se importante para os gestores entenderem o papel da internet e das redes sociais no contexto de envolvimento do consumidor a as dimensões que isso pode tomar em termos de fidelização. Além disso, é bem provável que a discussão sobre lealdade ainda continue viva por mais algumas décadas, considerando a complexidade do assunto e as 
infinitas possibilidades de abordagens que podem surgir com a evolução da sociedade e dos meios de consumo.

\section{MÉTODO}

Foram pesquisados artigos que analisaram a lealdade/fidelidade do consumidor. Utilizou-se artigos teóricos e teórico-empíricos, e aplicou-se, para estes tipos de artigos, os filtros de 27 revistas brasileiras. Os periódicos considerados estão indicados no Quadro 1. Decidiu-se ainda trabalhar com artigos publicados nos últimos 5 anos (2011 a 2015).

\section{Quadro 1: Lista de Periódicos Incluídos na Revisão}

\begin{tabular}{|c|c|}
\hline Periódicos Incluídos na Revisão & Qualis CAPES \\
\hline BAR - Brazilian Administration Review & $\mathrm{A} 2$ \\
\hline Organizações \& Sociedade & $\mathrm{A} 2$ \\
\hline Revis ta de Administração Contemporânea & $\mathrm{A} 2$ \\
\hline Revistas de Administração de Empresas & $\mathrm{A} 2$ \\
\hline Revista de Administração & $\mathrm{A} 2$ \\
\hline Revista de Administração Pública & $\mathrm{A} 2$ \\
\hline Cadernos EBRAPE & $\mathrm{A} 2$ \\
\hline Revista Brasileira de Gestão de Negócios & $\mathrm{A} 2$ \\
\hline Brazilian Business Review & B1 \\
\hline Gestão \& Produção & B1 \\
\hline Revis ta de Adminis tração Mackenzie & B1 \\
\hline Revista Eletrônica de Administração & B1 \\
\hline Revista de Gestão da Tecnologia e Sistemas de Informação & B1 \\
\hline Desenvolvimento em Questão - Unijuí & B2 \\
\hline Economia Global e Gestão & B2 \\
\hline Faces: Revista de Administração & B2 \\
\hline Gestão \& Regionalidade & B1 \\
\hline Organizações Rurais e Agroindustriais & B2 \\
\hline RAl: Revista de Administração e Inovação & B2 \\
\hline Revista Brasileira de Gestão e Desenvolvimento Regional & B2 \\
\hline Revis ta de Administração da Unimep & $\mathrm{B} 2$ \\
\hline Revista de Ciências da Administração & B2 \\
\hline Revista de Contabilidade e Organizações & B2 \\
\hline Revista Portuguesa e Brasileira de Gestão & B1 \\
\hline Revista de Administração da UFSM & B2 \\
\hline Revista Iberoamericana de Estratégia & $\mathrm{B} 2$ \\
\hline Revista Produção Online & B2 \\
\hline
\end{tabular}

Fonte: Elaborado pelos autores

A pesquisa dos artigos foi realizada na base de dados de cada uma das 27 revistas, utilizando-se para cada um destes quatro conjuntos de termos de busca bibliográfica: lealdade, fidelidade, loyalty e fidelity. Avaliou-se os títulos, resumos e metodologia dos artigos rejeitando-se os que não atenderam aos critérios determinados pela metodologia de busca. Encontrou-se no total 42 artigos que atenderam aos critérios de busca. 
Realizou-se a análise bibliométrica dos estudos selecionados, de acordo com os seguintes critérios: nome do artigo, autores, nome do periódico, Qualis Capes, ano de publicação, instituições participantes, enquadramento (teórico ou teórico-empírico), e, quando enquadrado como teórico-empírico: natureza do estudo, setor da economia, ramo de atuação, instrumentos de coleta de dados e técnicas de análise de dados.

\section{ANÁLISE DOS DADOS}

\subsection{ANÁLISE BIBLIOMÉTRICA}

A partir do levantamento dos 42 artigos encontrados nos 27 periódicos brasileiros elencados no Quadro 1, apresenta-se a síntese ilustrada dos resultados encontrados sobre lealdade e fidelidade do consumidor, elaborando-se ainda uma breve análise da produção nacional neste período.

Dos artigos pesquisados, a RAC lidera o número de publicações sobre lealdade e fidelidade do consumidor com seis artigos, representando 14,29\% do total. A RAE ficou em segundo lugar com cinco artigos publicados, representando 11,9\% do total. A Revista de Administração e a Revista Brasileira de Gestão de Negócios publicaram quatro artigos cada, representando 9,52\% do total cada uma. A Revista de Administração Mackenzie e a Revista de Ciências da Administração obtiveram 7,14\% do total com três artigos cada uma. Os periódicos BAR, BBR, Revista de Administração da UFSM, RAI e Revista Eletrônica de Administração apresentaram uma produção de $4,76 \%$ do total, com duas publicações cada. Por último, a Revista de Gestão da Tecnologia e Sistemas de Informação, FACES: Revista de Administração, Organizações \& Sociedades, Economia Global e Gestão, Revista Científica Eletrônica de Engenharia de Produção, Revista Ibero-Americana de Estratégia e Gestão \& Produção publicaram um artigo cada, com representação de $2,38 \%$ na produção total. A Figura 1 ilustra esses resultados.

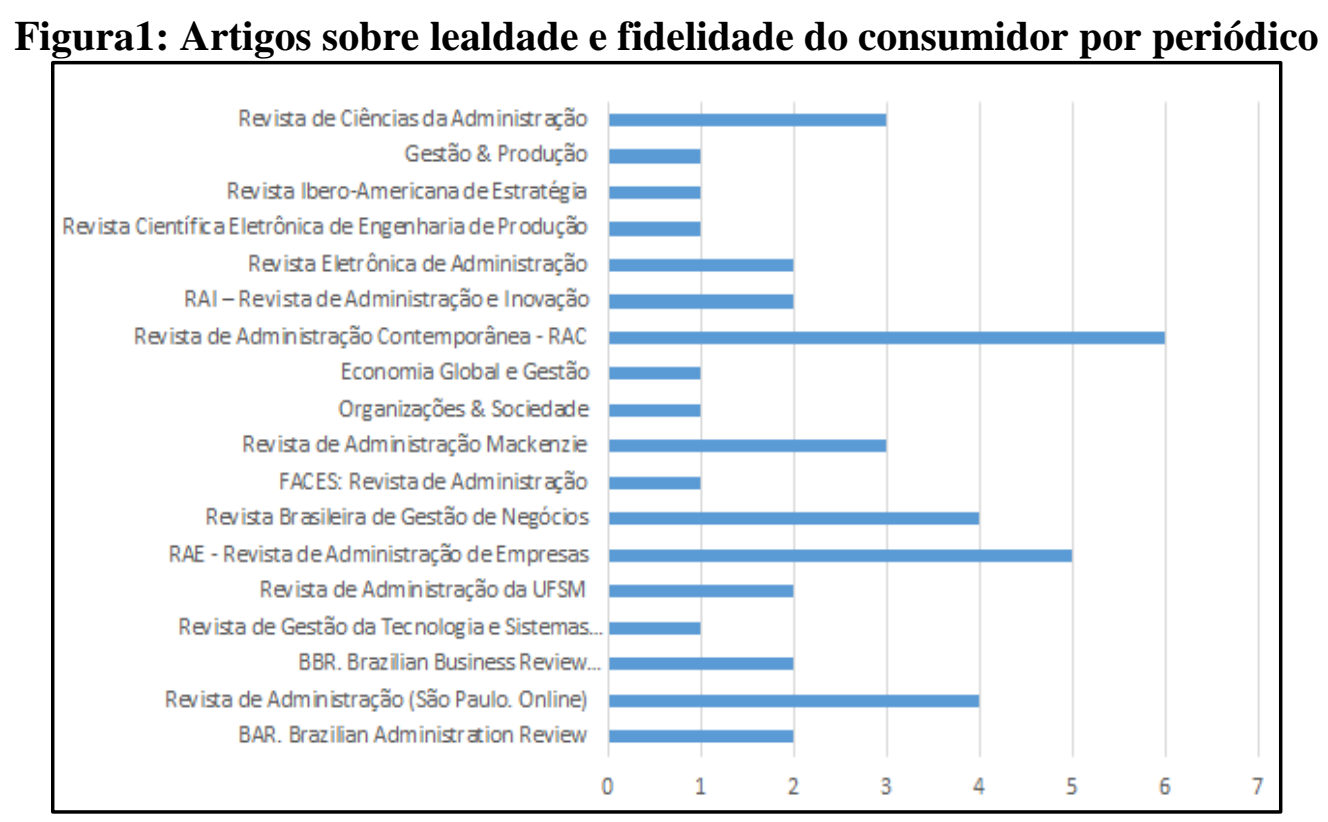

Fonte: Elaborado pelos autores

O período analisado foi produtivo com relação ao tema, apresentando uma média de 7 por ano. Verifica-se um pico de produção no ano de 2013, com 13 artigos publicados, representando 30,95\% do total. O segundo ano que mais produziu foi 2012 com 9 artigos, representando 21,43\% do total. Nos anos de 2011 e 2014 foram produzidos 8 artigos cada, 
representando 19,05\% do total cada ano. Os anos de 2010 e 2015 tiveram uma produção de 2 artigos cada, representando $4,76 \%$ do total. A Figura 2 apresenta os resultados.

Figura 2: Artigos sobre lealdade e fidelidade do consumidor por ano

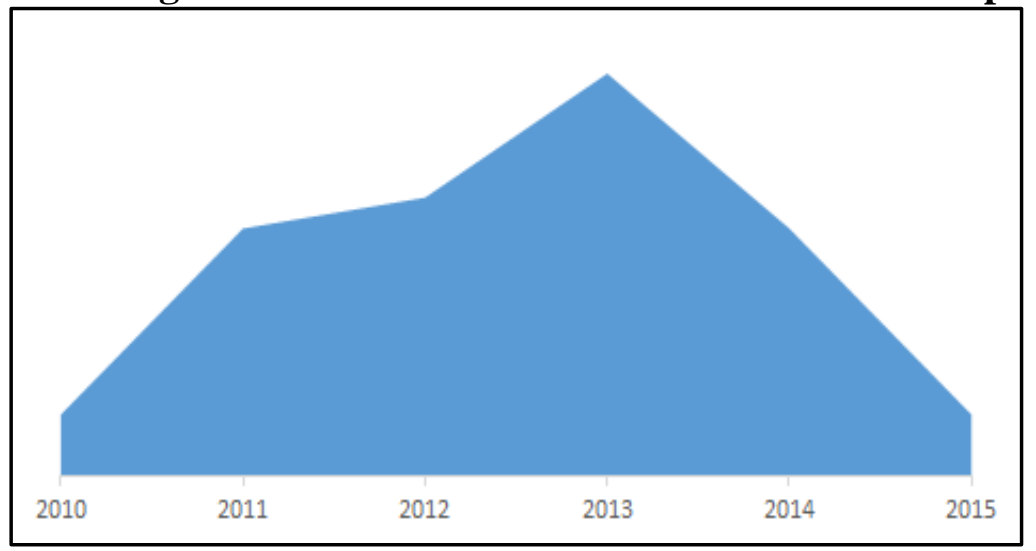

Fonte: Elaborado pelos autores

A maioria dos estudos enquadrou-se no modelo teórico-empírico, com 80,95\%, e apenas $19,05 \%$ dos trabalhos caracterizaram-se como ensaios teóricos. A Figura 3 apresenta a proporção encontrada quanto ao enquadramento dos estudos.

Figura 3: enquadramento dos estudos

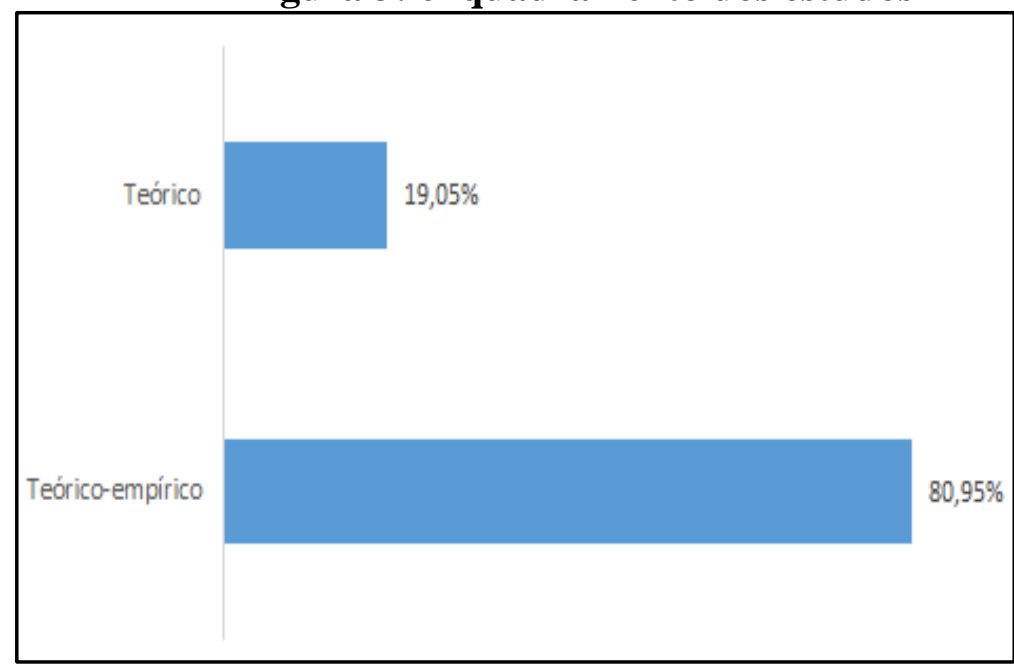

Fonte: Elaborado pelos autores.

Quanto às instituições de origem dos autores dos estudos, conforme mostra o Gráfico 4, observa-se que as instituições que mais publicaram artigos foram a Pontifícia Universidade Católica do Paraná (PUC/PR), Universidade de Fortaleza (UNIFOR) e Universidade de São Paulo (USP), com 7,61\% do total cada. Em segundo lugar no número de publicações está a Universidade Federal de Santa Catarina (UFSC) com 6,52\% do total. A Universidade Federal do Paraná (UFPR) e a Universidade Nove de Julho estão com 4,35\% cada uma. Empatadas com 3,26\% do total, estão: ESPM, FGV, FUMEC, Insper- Institute of Education and Research, UFES e UNIMEP. Com 2,17\% cada, aparecem: Faculdade Novos Horizontes, PUC/MG, UFMG, UFRGS, UnB e UNISINOS. As instituições com um artigo, representando 1,09\% cada, são apresentadas na Figura 4: 
Figura 4: Instituições de origem dos autores

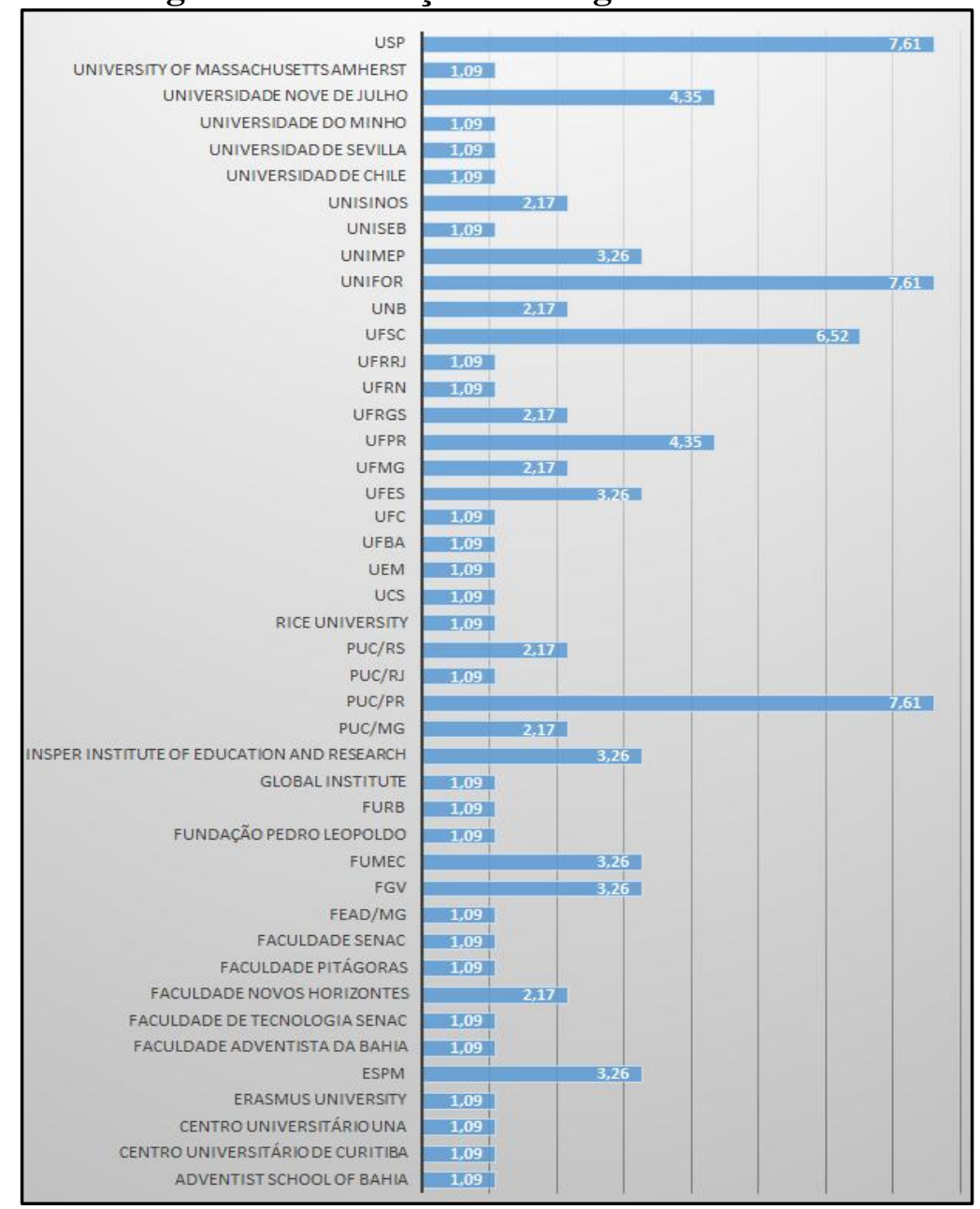

Fonte: Elaborado pelos autores.

Dentre os 42 trabalhos encontrados, alguns autores se destacaram ao publicarem mais de um artigo dentro do período estudado. Destacam-se Eliane Cristine Francisco-Maffezzolli (PUC/PR), Evandro Luiz Lopes (UNIVERSIDADE NOVE DE JULHO) e Paulo Henrique Muller Prado (UFPR) com três artigos cada. Ressalta-se que os demais autores relatados publicaram um total de dois artigos cada um. O Quadro 2 apresenta o nome de cada um desses autores e suas instituições de origem. 
Quadro 2: Autores que mais publicaram no período estudado

\begin{tabular}{|l|c|}
\hline \multicolumn{1}{|c|}{ AUTOR } & N DE ARTIGOS \\
\hline Evandro Luiz Lopes - UNIVERSIDADE NOVE DE JULHO & 3 \\
\hline Eliane Cristine Francisco-Maffezzolli - PUC/PR & 3 \\
\hline Paulo Henrique Muller Prado - UFPR & 3 \\
\hline Clara Márcia Ribeiro - FACULDADE PITÁGORAS & 2 \\
\hline Elder Semprebom - PUC/PR & 2 \\
\hline Braulio Oliveira - USP & 2 \\
\hline Antonio Carlos Giuliani - UNIMEP & 2 \\
\hline Fábio Vinicius de Macedo Bergamo - ADVENTIST SCHOOL OF BAHIA & 2 \\
\hline Maria Aparecida Gouvêa - USP & 2 \\
\hline Mateus CanniattiPonchio - ESPM & 2 \\
\hline Paulo de Paula Baptista - PUC/PR & 2 \\
\hline Celso Augusto de Matos - UNISINOS & 2 \\
\hline Plínio Rafael Reis Monteiro - UFMG & 2 \\
\hline Sandra Sayuri Yamashita Nagakawa - USP & 2 \\
\hline
\end{tabular}

Fonte: Elaborado pelos autores

Dentre os 42 artigos encontrados, o estudo relacional foi o tema mais estudado com uma representação de $33,33 \%$ do total. O assunto desenvolvimento e validação de medidas apresentou uma contribuição de $30,95 \%$ do total. Conceitos e fundamentos ficou em terceiro lugar com 19,05\% do total, seguindo de variáveis correlatas com 11,9\% do total. Por último, com $4,76 \%$ de representação, encontra-se a categoria tecnologia e serviços de informação. Os resultados podem ser visualizados na Figura 5:

Figura 5: Assuntos mais estudados

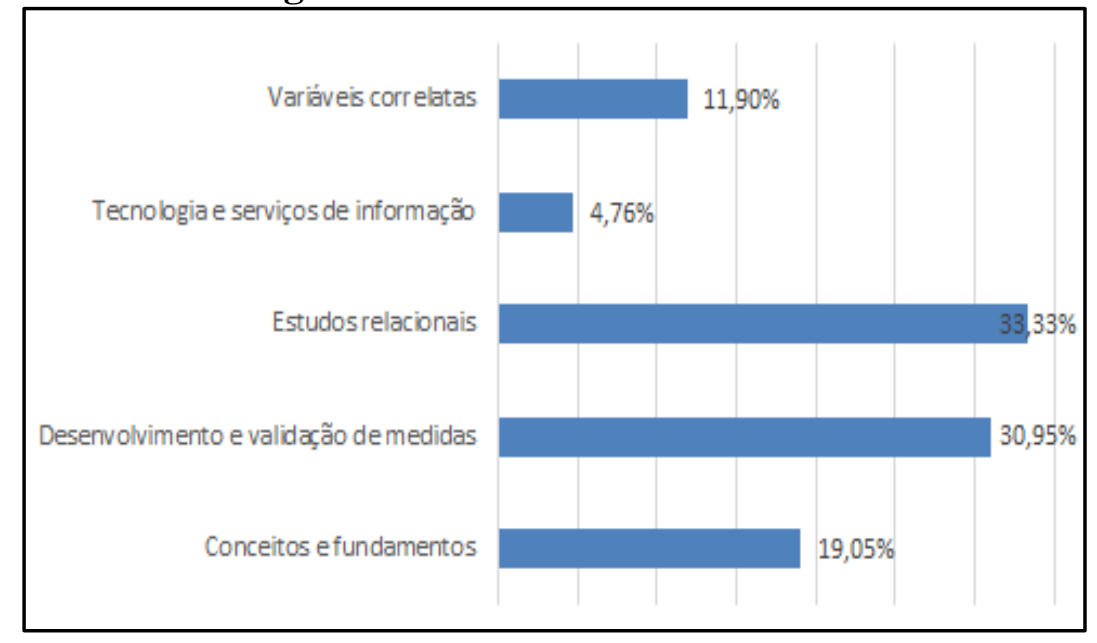

Fonte: Elaborado pelos autores.

GєCont, v. 3, n. 2, Floriano-PI, Jul-Dez. 2016. 
Dos 42 trabalhos selecionados, 34 são artigos teórico-empíricos. Entre eles, há uma predominância de estudos quantitativos (22 artigos), representando $64,71 \%$ do total. Foram encontrados quatro artigos de natureza qualitativa representando $11,76 \%$ do total e oito de natureza quali-quanti, representando $23,53 \%$ do total. Verifica-se na Figura 6 a predominância de estudos de natureza quantitativa.

Figura 6: Natureza dos artigos teórico-empíricos

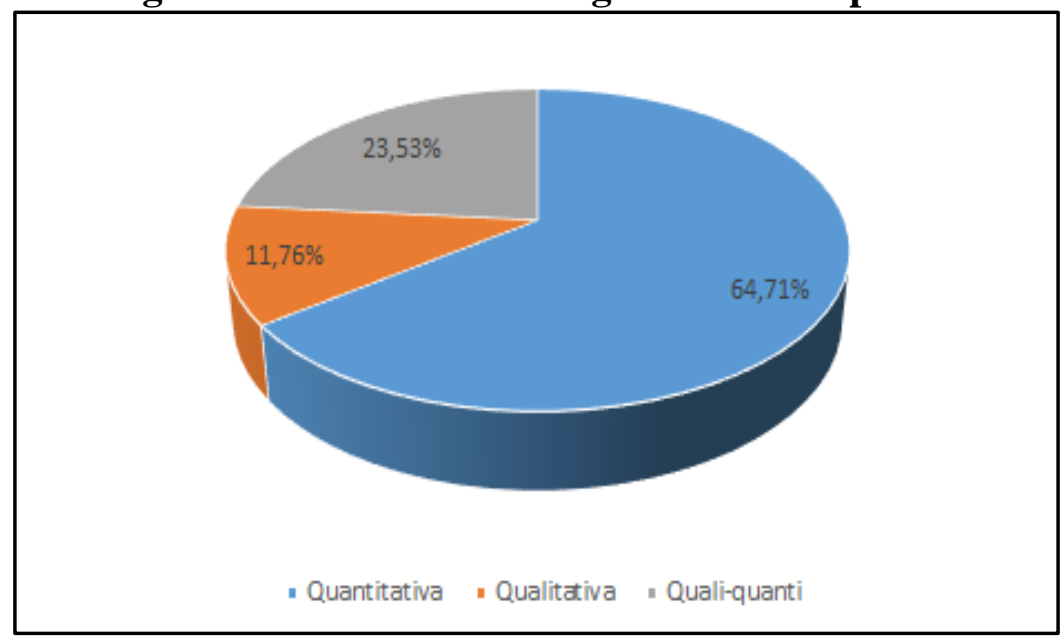

Fonte: Elaborado pelos autores

No que tange aos estudos teórico-empíricos encontrados, todos foram desenvolvidos em empresas do setor privado (segundo setor). Não foi encontrado nenhum artigo com o primeiro ou terceiro setores da economia que trata-se do tema lealdade ou fidelidade do consumidor.

As empresas participantes dos estudos distribuem-se nos mais diversos ramos de atuação. O maior foco dos pesquisadores esteve no setor varejista, responsável por 20,59\% do total. Estudos realizados no setor de comércio eletrônico, tecnologia da informação e telecomunicações corresponderam a 11,76\% do total cada. Em terceiro lugar, encontram-se os ramos de esportes com 8,82\% do total. Empatados, com 5,88\% cada, aparecem os setores bancário, educação, transporte e turismo. Com 2,94\% cada, aparecem os seguintes setores: alimentação, editorial, hospitalar e música. Os dados com relação ao ramo das empresas estudadas podem ser verificados na Figura 7: 
Figura 7: Ramo de atuação das empresas pesquisadas

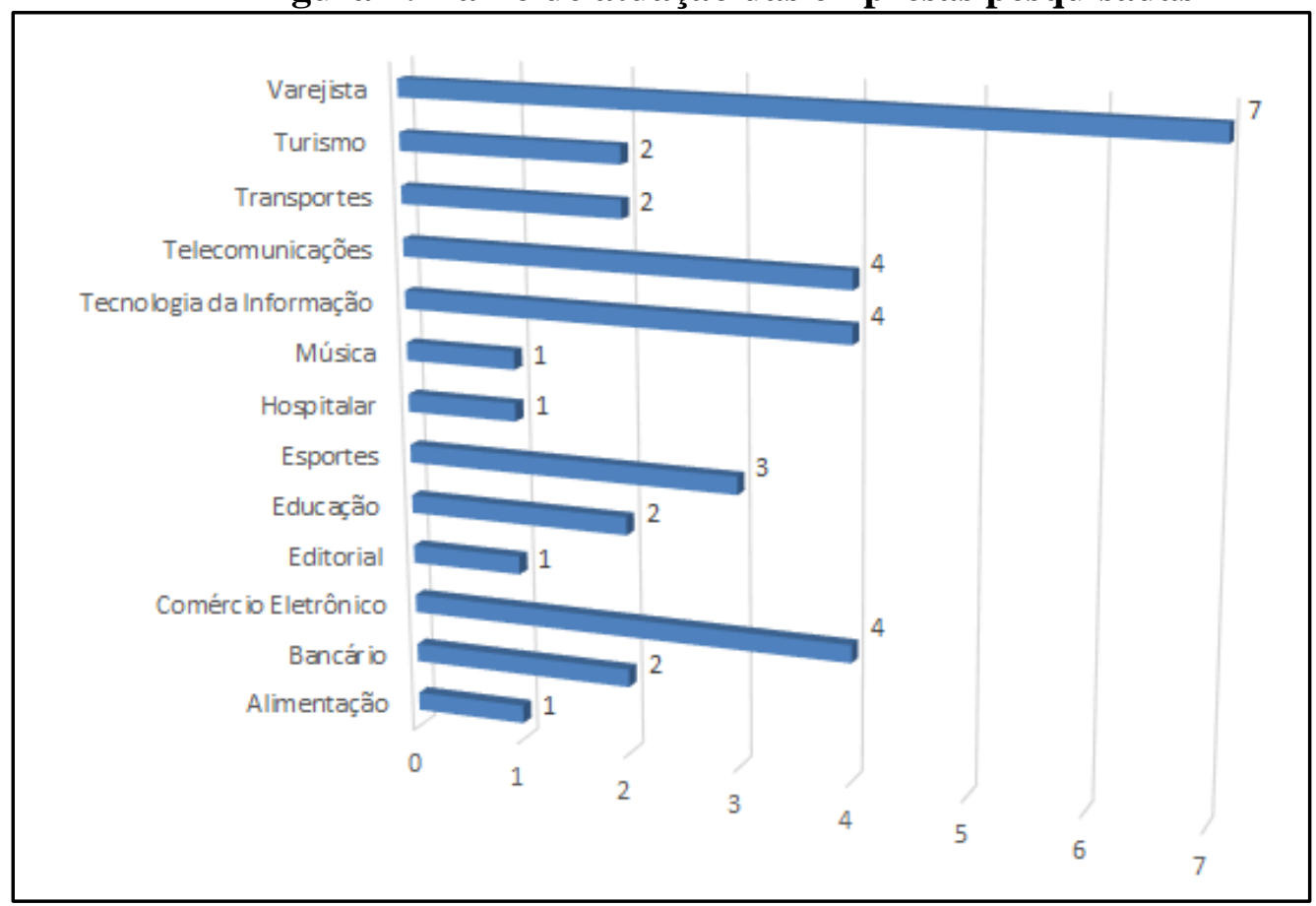

Fonte: Elaborado pelos autores

Verificou-se que $73,53 \%$ do total dos estudos utilizaram o questionário como meio para coleta de dados, o que condiz com a predominância de artigos de natureza quantitativa. Em segundo lugar, com 8,82\% do total, encontra-se a entrevista. Em terceiro lugar, os métodos mais utilizados foram o estudo de caso e o grupo focal, representando 5,58\% do total das pesquisas cada. E, em último, estão a netnografia e a observação com $2,94 \%$ do total cada. Os dados referentes aos principais métodos de coleta encontram-se na Figura 8:

Figura 8: Instrumentos de coletas de dados utilizados

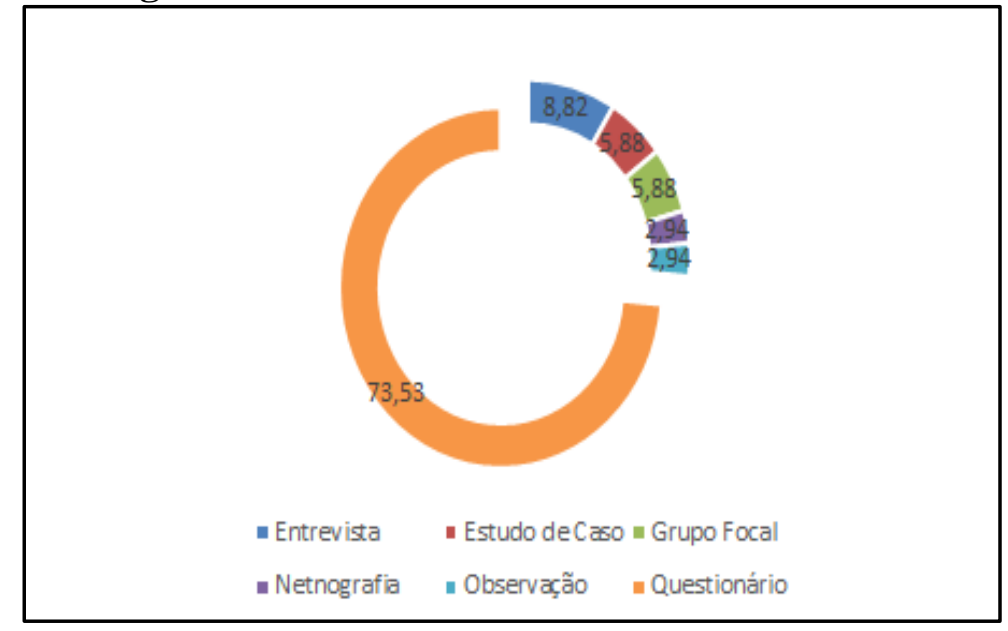

Fonte: Elaborado pelos autores

Quanto à técnica de análise dos dados, $50 \%$ dos estudos desenvolvidos no período pesquisado utilizaram equações estruturais. A estatística descritiva foi utilizada para análise de $26,47 \%$ do total de artigos, seguido pela análise de regressão múltipla com $17,65 \%$. A análise de conteúdo representa $5,88 \%$ do total dos estudos. Esses resultados corroboram com os achados do Gráfico 6, onde a maioria dos estudos foi de natureza quantitativa. As 
informações sobre as técnicas utilizadas pelos pesquisadores para a análise dos dados estão discriminadas na Figura 9.

Figura 9: Métodos de análises mais utilizados

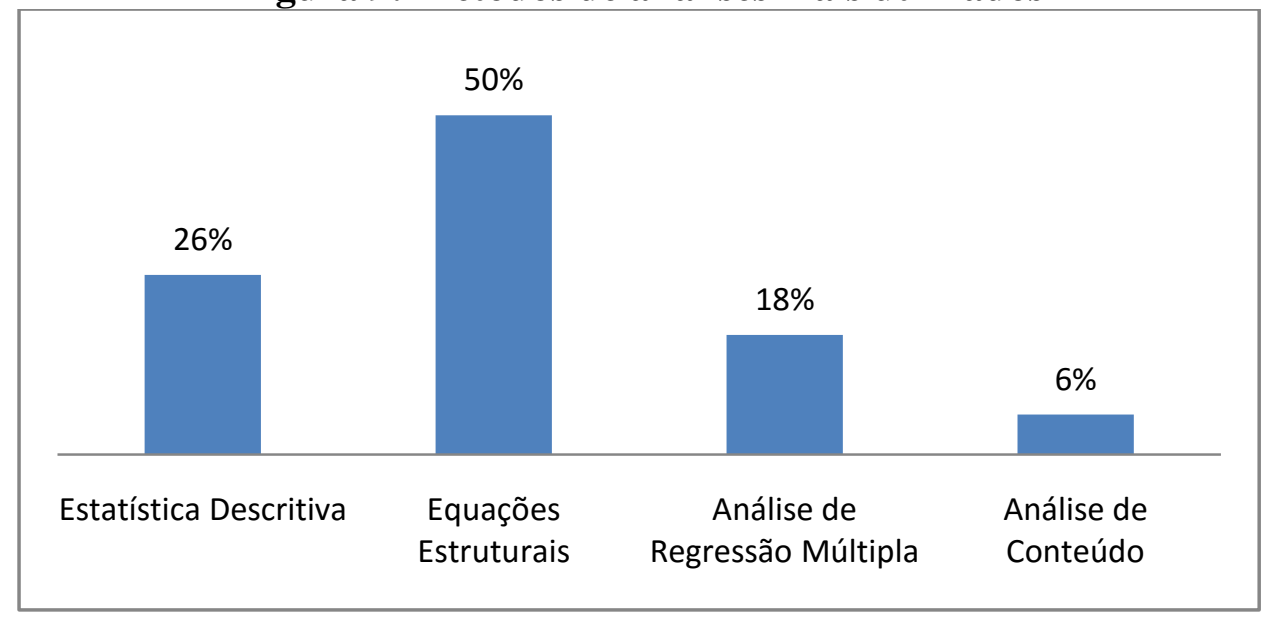

Fonte: Elaborado pelos autores

\subsection{DISCUSSÃO E AGENDA DE PESQUISA}

A partir das informações obtidas na seção de produção nacional, é possível traçar um panorama a respeito da evolução dos estudos sobre lealdade e fidelidade do consumidor no país.

Comparando-se aos estudos realizados em outros países, abordados na seção "estado da arte", no Brasil ainda é possível perceber uma grande concentração nos estudos voltados para os grandes varejistas e focados no consumo de produtos.

Como proposta de agenda de pesquisa indica-se a necessidade de estudos voltados para a indústria de serviços, que tem se tornado foco internacionalmente e que, inclusive, é um dos principais focos da economia nacional, uma vez que o país não tem tradição na indústria de manufatura se comparado com países como os EUA.

Outra vertente de estudos que ganha força internacionalmente e que pode ser mais abordada internamente é a do mundo virtual e seus impactos no consumo e na fidelização. Além do comércio eletrônico em si, tendo aparecido em segundo lugar como ramo de atuação dos estudos nacionais, é interessante aumentar a abrangência das análises envolvendo também aspectos, como, por exemplo, o impacto de redes sociais no consumo e na lealdade do consumidor. A tendência percebida é que os estudos acompanhem as mudanças sociais que podem influenciar no tema de interesse, que no caso é a lealdade.

Em relação à abordagem utilizada, há ainda muito espaço para o desenvolvimento de pesquisas qualitativas e quali-quanti. Entende-se aqui que realidades locais podem ser interessantes para a compreensão do fenômeno como um todo e que os instrumentos adotados em trabalhos com enfoque quantitativo, em sua maioria de origem internacional, podem não representar tão bem a realidade do consumidor brasileiro.

Dito isso, sugere-se que, em estudos futuros, os pesquisadores optem por abordagem técnica e instrumento de coleta que possibilitem entendimento mais aprofundado da realidade nacional, onde abordagens qualitativas ganham conotação especial. Por exemplo, uma pesquisa etnográfica poderia ser realizada para entender melhor as nuances da relação entre o consumidor brasileiro e as marcas, bem como em que situações facilitam ou dificultam o surgimento de uma relação de fidelidade. 


\section{CONSIDERAÇÕES FINAIS}

Este trabalho procurou contribuir com a produção acadêmica no campo de conhecimento teórico sobre a fidelidade e a lealdade do consumidor, ao realizar uma síntese da literatura (estado da arte) e uma revisão da produção nacional.

Ressalta-se que esta pesquisa não pretendeu esgotar a literatura nacional concernente ao assunto no período investigado, uma vez que restringiu-se à pesquisa aos periódicos científicos da área de Administração, apresentados no Quadro 1. A ideia foi realizar um mapeamento sobre a produção científica brasileira recente de primeira linha a fim de embasar novas pesquisas futuras a respeito do tema.

Entende-se que, embora as pesquisas teórico-empíricas de natureza quantitativa tenham se destacado, a interpretação garantida pela pesquisa qualitativa é de fundamental importância para o desenvolvimento de novos estudos sobre lealdade de clientes, propondo novas teorias e paradigmas que possam contribuir para a construção de um arcabouço teórico mais consistente a fim de promover o avanço do conhecimento nesta área.

\section{REFERÊNCIAS}

CASALÓ, L.V.; FLAVIÁN, C.; GUINALÍU, M. Relationship, community promotion and brand loyalty in virtual communities: Evidence from free software communities. International Journal of Information Management, 30, 357-367, 2010.

ČATER, T.; ČATER, B. Product and relationship quality influence on customer commitment and loyalty in B2B manufacturing relationships. Industrial Marketing Management, 39, 1321-1333, 2010.

CHEN, P-T.; HU, H-H. The effect of relational benefits on perceived value in relation to customer loyalty: An empirical study in the Australian coffee outlets industry.

International Journal of Hospitality Management, 29, 405-412, 2010.

COELHO, P. S.; HENSELER, J. Creating customer Loyalty through service customization. European Journal of Marketing, Vol. 46, No. 3/4, pp. 331-356, 2012.

FOXALL, G. R., OLIVEIRA-CASTRO, J. M., JAMES, V. K., YANI-DE-SORIANO, M. M., \& SIRGUDSSON, V. Consumer behavior analysis and social marketing: The case of environmental conservation. Behavior and Social Issues, 15, 101-124, 2006.

GREMLER, Dwayne; BROWN, Stephen. The loyalty-ripple effect: appreciating the full value of customers. International Journal of Service Industry Management, v. 10, n. 3, p. 271-291, 1999.

KRYSTALLIS, A.; CHRYSOCHOU, P. The effects of service brand dimensions on brand loyalty. Journal of Retailing and Consumer Services, 21, 139-147, 2014.

LAROCHE, M.; HABIBI, M. R.; RICHARD, M-O.; SANKARANARAYANAN, R. The effects of social media based brand communities on brand community markers, value creation practices, brand trust and brand loyalty. Computers in Human

Behavior, 28, 1755-1767, 2012. 
SETÓ-PAMIES, D. Customer loyalty to service providers: examining the role of service quality, customer satisfaction and trust. Total Quality Management \& Business Excellence, 23:11-12, 1257-1271, 2012.

SHARP, B.; SHARP, A. Loyalty Programs and Their Impact on Repeat Purchase Loyalty Patterns. International Journal of Research in Marketing, 14, 473-486, 1997.

SIRDESHMUKH, D., SINGH, J., \& SABOL, B. Consumer trust, value, and loyalty in relational exchanges.Journal of Marketing, 66(1), 15-37, 2002.

TOUFAILY, E; RICARD, L.; PERRIEN, J. (). Customer loyalty to a commercial website: Descriptive meta-analysis of the empirical literature and proposal of an integrative model.Journalof Business Research,2012.

UNCLES M., EHRENBERG A., HAMMOND K. Patterns of buyer behavior: regularities, models and extensions. Marketing Science, 14, G71-G78, 1995.

WELLS, V. K.; FOXALL, G. R. Matching, demand, maximization and consumer choice. Psychological Record, v. 63, n. 2, p. 239-258, 2013.

ZEITHMAL, V. A., BERRY, L. L., \& PARASURAMAN, A. The behavioral consequences of service quality. Journal of Marketing. 60(2), 31-47, 1996. 